\title{
Fenestration of the vertebrobasilar junction detected with multidetector computed tomography angiography
}

\author{
B.R. Omotoso ${ }^{1}$, R. Rarrichandparsad ${ }^{2}$, I.G. Moodley ${ }^{\circledR}$, K.S. Satyapal' ${ }^{1}$, L. Lazarus ${ }^{1}$ \\ ${ }^{1}$ Discipline of Clinical Anatomy, School of Laboratory Medicine and Medical Sciences, College of Health Sciences, \\ University of KwaZulu-Natal, Westville Campus, Durban, South Africa \\ 2Department of Neurosurgery, School of Clinical Medicine, College of Health Sciences, Nelson R Mandela School \\ of Medicine, University of KwaZulu-Natal, Durban, South Africa \\ ${ }^{3}$ Department of Radiology, Jackpersad and Partners Inc., Specialist Diagnostic Radiologists, Lenmed Ethekwini Hospital \\ and Heart Centre, South Africa
}

[Received: 5 June 2020; Accepted: 20 July 2020; Early publication date: 2 March 2021]

\begin{abstract}
The complex embryonic origin of the vertebrobasilar system may result in a wide range of anatomical variations. It has been hypothesized that the formation of fenestrations are likely to occur due to the failure of regression of the bridging arteries that connect the longitudinal neural arteries during embryogenesis. Fenestration of the vertebrobasilar system is a rare anatomical variation that involves a luminal division of the artery, that has a single origin into two separate and parallel channels which are rejoined distally. Fenestrations are important anatomical variants in patients undergoing endovascular and invasive intracranial interventions. Vascular fenestration has been associated with aneurysms, arteriovenous malformations, neuralgia, and vertebrobasilar ischaemia. We report on 3 cases of fenestration at the vertebrobasilar junction in 1 female and 2 male patients, respectively, using multidetector computed tomography angiography. The length of the fenestrated segment of the artery measured $4.41 \mathrm{~mm}, 3.90 \mathrm{~mm}$, and $5.90 \mathrm{~mm}$, respectively in the patients. Our report is clinically important as the presence of this anatomical variation may influence the management of cervical and intracranial pathologies. Increased awareness of the prevalence of anatomic variations contributes to the advancement of noninvasive imaging capabilities. (Folia Morphol 2022; 81, 2: 510-514)
\end{abstract}

Key words: morphological variation, vertebral artery, basilar artery

\section{INTRODUCTION}

The vertebrobasilar system is the combination of the two vertebral arteries (VAs) together with the basilar artery. The vertebrobasilar junction (VBJ) is the point of union of the bilateral VAs, mostly at the lower border of the pons [19] to form the basilar artery. The vertebrobasilar system provides the blood supply to vital structures like the cervical spinal cord, brainstem, thalamus, cerebellum, and occipital lobes [18]. Therefore, a morphological variation of either of the VA or basilar trunk can influence vascular supply to the afore mentioned structures. Anatomically, VAs are divided into four segments: prevertebral (V1), vertebral (V2), atlantoaxial (V3), and intracranial (V4) segments [1]. Pre-

Address for correspondence: Prof. L. Lazarus, Department of Clinical Anatomy, School of Laboratory Medicine and Medical Sciences, College of Health Sciences, University of KwaZulu-Natal, Private Bag X54001, Durban, 4000, South Africa, tel: +27 31 260 7899, e-mail: ramsaroopl@ukzn.ac.za

This article is available in open access under Creative Common Attribution-Non-Commercial-No Derivatives 4.0 International (CC BY-NC-ND 4.0) license, allowing to download articles and share them with others as long as they credit the authors and the publisher, but without permission to change them in any way or use them commercially. 
Table 1. Incidence of vertebrobasilar junction (VBJ) fenestration in different population groups

\begin{tabular}{lccccc}
\hline Author (year) & Country & Type of study & $\begin{array}{c}\text { Number } \\
\text { of cases }\end{array}$ & $\begin{array}{c}\text { Sex: } \\
\text { male/female }\end{array}$ & $\begin{array}{c}\text { Segment of } \\
\text { vertebral arteries }\end{array}$ \\
\hline Campos et al. (1987) [2] & Canada & DSA & 21 & $7 / 14$ & VBJ \\
Yoon et al. (2004) [24] & South Korea & CTA & 4 & $2 / 2$ & VBJ \\
Consoli et al. (2013) [3] & Italy & DSA & 2 & Female & VBJ \\
Kan et al. (2013) [9] & United States & DSA & 1 & Female & VBJ \\
Gupta et al. (2013) [7] & India & CTA, DSA & 4 & $3 / 1$ & VBJ \\
Trivelato et al. (2016) [22] & Brazil & DSA & 5 & $1 / 4$ & VBJ \\
Zhu et al. (2016) [25] & China & DSA & 10 & $6 / 4$ & VBJ \\
Present study & South Africa & CTA & 3 & $1 / 2$ & VBJ
\end{tabular}

CTA — computed tomography angiography; DSA — digital subtraction angiography

vious studies regarding the morphology of the VA have shown the existence of variation in its course $[5,6,11]$.

Fenestration is an uncommon anatomical variation that involves a luminal division of an artery that has a common origin into two separate and parallel channels anywhere along its course to rejoin distally. Fenestration is sometimes mistaken for duplication, but should, however, be differentiated. Duplication normally involves double vascular origin which later fuse during the course of the artery [8].

Fenestration of the VBJ has been reported to be a predisposing factor to other vascular malformations like aneurysms [24], arteriovenous malformations [12], and neuralgia [10]. Furthermore, fenestration has been associated with epidermoid cysts and vertebrobasilar ischaemia [13, 23]. A thorough understanding of the anatomy and knowledge of anatomical variations of the vertebrobasilar artery is essential for assessing neurologic syndromes and preoperative neurosurgical planning. In this report, we describe three cases of VBJ fenestration using multidetector computed tomography angiography.

Fenestration of the VBJ is a morphological variation that has been previously reported in the international population, for example in China, United States, Canada and Brazil (Table 1). In this report, we present 3 cases of fenestration of the VBJ in 2 White and 1 Indian South Africans.

\section{CASE REPORT}

Our study is retrospective in nature, therefore, written informed consent was not obtained. However, the design of this study was approved by our Institutional Review Board/Ethics Committee (Biomedical Research Ethics Committee of the University of Kwa-
Zulu-Natal with ethical No: BE 148/19). No identifying patient information is present in this paper.

\section{Case 1}

Computed tomography angiography (CTA) scan of intracranial vessels of a 34-year-old Indian South African female at Lenmed Ethekwini Hospital and Heart Centre illustrated fenestration at the VBJ (Fig. 1A). The length of the fenestrated segment at the VBJ was $4.41 \mathrm{~mm}$ with the two limbs having a similar diameter. Clinical examination showed extensive aneurysmal subarachnoid haemorrhage in the right Sylvian fissure with intracerebral extension into the adjacent peri-lenticular parenchyma. Ruptured right middle cerebral artery saccular aneurysm, and further multilobulated anterior cerebral artery aneurysm was noted with the incorporation of large left A3 segment.

\section{Case 2}

A 79-year-old male White South African presented to Lenmed Ethekwini Hospital and Heart Centre for the history of vertebrobasilar transient ischaemic attack (TIA) and acute unsteadiness. CTA scan revealed fenestration at the VBJ (Fig. 1B). The length of the fenestrated segment at the VBJ was $3.90 \mathrm{~mm}$ with the two limbs having a similar diameter. The posterior communicating artery was hypoplastic on the right when compared to the left and no intracranial aneurysm was demonstrated.

\section{Case 3}

An 83-year-old male White South African presented to Lenmed Ethekwini Hospital and Heart Centre for a clinical history of collapse, recurrent TIA, ataxia, and diplopia. CTA showed fenestration at the VBJ 



Figure 1. Computed tomography angiography images of case 1 (A), case 2 (B) and case 3 (C); A. Three-dimensional reconstructed image shows vessels of anterior and posterior circulation. Blue arrow shows intracranial segment of right vertebral artery (VA) while the red arrow shows intracranial segment of the left VA. The white arrow shows fenestration at the vertebrobasilar junction (VBJ) between the confluence of bilateral VAs and proximal part of the basilar artery; B. Three-dimensional reconstructed image shows the intracranial VA, bending basilar artery and the circle of Willis. The white arrow shows fenestration at the VBJ while the yellow arrow shows the basilar artery; C. Three-dimensional reconstructed image shows the intracranial VA, bending basilar artery and the circle of Willis. The white arrow shows fenestration at the VBJ while the blue arrow shows the basilar artery.

(Fig. 1C). The length of the fenestrated segment at the VBJ was $5.90 \mathrm{~mm}$ with the two limbs having a similar diameter. The basilar artery was minimally ecstatic involving the medial and lateral margin at its bifurcation.

\section{DISCUSSION}

Anatomical variation in the origin of the VA is the most reported morphological variation. This has also been reported in the South African population $[4,15]$. However, reports on fenestration at the VBJ are scarce and are mostly reported internationally (Table 1). Previous studies regarding the morphology of the VA have shown the existence of variation with demographic and ethnic/racial differences $[5,6,11]$. Complex embryogenesis of the vertebrobasilar system results in vascular fenestration and it is frequently reported at the extracranial portion of the VA [12]. In contrast, the three cases we report showed fenestration at the VBJ.

Embryologically, the VA is formed during the $32^{\text {nd }}$ to $40^{\text {th }}$ days by the development of longitudinal anastomosis between the seven adjacent cervical intersegmental arteries that are formed from the primitive dorsal aorta [17]. Later, however, the first six cervical intersegmental arteries regress, while the seventh persists to form the proximal part of the subclavian artery and the point of origin of the adult VA. The basilar trunk is formed from the fusion of primitive embryonic longitudinal neural arteries by approximately the fifth foetal week [14]. Generally, fenestration at any of the segments of the VA (V1-V4) is due to the absence of obliterations of two intersegmental vessels which fuse, or by segmental arteries which become short or disappear while a portion of the dorsal aorta remains against the VA [1]. Basilar artery fenestration occurs as a result of partial failure or incomplete fusion of the longitudinal neural arteries and regression of the bridging arteries connecting the longitudinal arteries [25]. Although fenestration at the VBJ is formed between the confluence of the bilateral distal VA and proximal part of the basilar artery, researchers agree that the best explanation for this morphological variation is the persistence or incomplete fusion of one of the temporary bridging arteries between the embryologic bilateral longitudinal neural arteries that form the basilar artery $[7,25]$. This could occur as a result of some genetic or environmental factors [25]. In the cases we observed, fenestration was found at the VBJ in 3 patients (Figs. $1 A-C$ ).

The macroscopic examination of the fenestrated segment revealed a unique fusiform thickened vessel [1]. Furthermore, microscopic and histopathological examination of the limbs has shown irregularities in the lateral and medial wall structure $[1,13]$. These 
irregularities may alter the haemodynamics of blood flow at the proximal and distal end of the fenestrated segment causing TIA as reported in two of our patients (cases 2 and 3 ).

Vertebral artery dominance and bending of the basilar artery have also been reported as risk factors for brainstem infarction and TIA [16]. Morphologically, the VBJ is subjected to the greatest stresses of flow and turbulence from the bilateral VA below. This complex geometry of the $\mathrm{VBJ}$, in addition to fenestration and basilar bends (Fig. 1A-C), may also contribute to the clinical history of TIA in cases 2 and 3 .

Some authors suggest that fenestration of the VA is an incidental finding and has no pathological and clinical importance [20], while others hypothesized that its occurrence increases the prevalence of aneurysms (most especially at the VBJ) [2, 24]. Fenestration has also been associated with the brain, spinal cord, and spinal column abnormalities in addition to other vascular disorders [21]. In case 1, there is a middle cerebral artery and anterior cerebral artery aneurysm, which is not associated with the fenestration. According to the report by Campos et al. [2] and review of literature (Table 1), fenestration at the VBJ is more common in female subjects while VA fenestration is considered to be more frequent in male subject [12]. This may not be comparable due to different embryological processes in the formation of the basilar artery as opposed to the vertebral arteries. Also, our report is a small series with only 3 subjects comprising 2 males and 1 female.

\section{CONCLUSIONS}

The authors presented 3 cases of fenestration at the VBJ in 2 White males and 1 female Indian South African patients. The presence of this morphological variation can increase the incidence of an aneurysm. Since most intracranial vascular disorders such as aneurysm are treated using endovascular procedures, a knowledge of the presence of fenestrations may influence the endovascular strategy. Therefore, knowledge of this anatomical variation will help in the preoperative workup and contribute to the correct interpretation of preoperative images. This report will also contribute to the demography of the South African population.

Conflict of interest: None declared

\section{REFERENCES}

1. Bruneau M, De Witte O, Regli L. Anatomical variations, in Pathology and surgery around the vertebral artery. Springer Paris, Paris 2011: 53-74.

2. Campos J, Fox A, Vinuela F. Saccular aneurysms in basilar artery fenestration. AJNR Am J Neuroradiol. 1987; 8(2): 233-236.

3. Consoli A, Renieri L, Nappini S, et al. Endovascular treatment with 'kissing' flow diverter stents of two unruptured aneurysms at a fenestrated vertebrobasilar junction. J Neurointerv Surg. 2013; 5(2): e9, doi: 10.1136/neurintsurg-2011-010188, indexed in Pubmed: 22315285.

4. Da Silva R. Anatomical study of the variation in the branching patterns and histology of the aorta in a South African population. University of Cape Town 2013.

5. Deng D, Cheng FuBo, Zhang Y, et al. Morphological analysis of the vertebral and basilar arteries in the Chinese population provides greater diagnostic accuracy of vertebrobasilar dolichoectasia and reveals gender differences. Surg Radiol Anat. 2012; 34(7): 645-650, doi: 10.1007/ s00276-012-0960-9, indexed in Pubmed: 22427028.

6. Gaigalaite V, Vilimas A, Ozeraitiene V, et al. Association between vertebral artery hypoplasia and posterior circulation stroke. BMC Neurol. 2016; 16: 118, doi: 10.1186/ s12883-016-0644-x, indexed in Pubmed: 27461465.

7. Gupta V, Ahuja CK, Khandelwal N, et al. Treatment of ruptured saccular aneurysms of the fenestrated vertebrobasilar junction with balloon remodeling technique. A short case series and review of the literature. Interv Neuroradiol. 2013; 19(3): 289-298, doi: 10.1177/159101991301900305, indexed in Pubmed: 24070077.

8. Ionete $\mathrm{C}$, Omojola MF. MR angiographic demonstration of bilateral duplication of the extracranial vertebral artery unusual course and review of the literature. AJNR Am J Neuroradiol. 2006; 27(6): 1304-1306.

9. Kan P, Abla AA, Dumont TM, et al. Double-barrel stent-assisted coiling of a basilar artery fenestration aneurysm. J Neuroimaging. 2013; 23(3): 496-499, doi: 10.1111/j.1552-6569.2012.00720.x, indexed in Pubmed: 22607550.

10. Kim K, Mizunari T, Kobayashi S, et al. [Occipital neuralgia caused by the compression of the fenestrated vertebral artery: a case report]. J Neurosurg. 1999; 27(7): 645-650, indexed in Pubmed: 10440039.

11. Kornieieva M, Al-Hadidi A. Morphology of the vertebral artery in Asian population. Asian J Med Sci. 2014; 5(4): 84-88, doi: 10.3126/ajms.v5i4.6150.

12. Kowada M, Yamaguchi K, Takahashi H. Fenestration of the vertebral artery with a review of 23 cases in Japan. Radiology. 1972; 103(2): 343-346, doi: 10.1148/103.2.343, indexed in Pubmed: 5024559.

13. Kubo M, Hacein-Bey L, Varelas PN, et al. Ruptured saccular aneurysm of distal vertebral artery fenestration managed with Guglielmi detachable coils and intraventricular tissue plasminogen activator. Surg Neurol. 2005; 63(3): 244-248, doi: 10.1016/j.surneu.2004.02.038, indexed in Pubmed: 15734513. 
14. Luh GY, Dean BL, Tomsick TA, et al. The persistent fetal carotid-vertebrobasilar anastomoses. AJR Am J Roentgenol. 1999; 172(5): 1427-1432, doi: 10.2214/ ajr.172.5.10227532, indexed in Pubmed: 10227532.

15. Makhanya NZ, Mamogale RT, Khan N. Variants of the left aortic arch branches. SA J Radiol. 2004; 8(4): 10, doi: 10.4102/sajr.v8i4.102.

16. Meng $X$, Ding $W, W u X$, et al. Clinical investigation and characterization of vertebrobasilar dolichoectasia and vertebral artery dominance. Discov Med. 2018; 25(138): 151-158, indexed in Pubmed: 29723487.

17. Padget $\mathrm{DH}$. The development of the cranial arteries in the human embryo. J Contrib Embryol. 1948; 32: 205-261.

18. Piccinin MA, Munakomi S, Neuroanatomy, Vertebrobasilar System, in StatPearls [Internet]. StatPearls Publishing, 2019.

19. Standring S. Gray's anatomy e-book: the anatomical basis of clinical practice. Elsevier Health Sciences 2015.

20. Tetiker H, Cimen M, Koşar MI. Fenestration of the vertebral artery: case presentation. Folia Morphol. 2014; 73(1): 84-86, doi: 10.5603/FM.2014.0012, indexed in Pubmed: 24590528
21. Tran-Dinh HD, Soo YS, Jayasinghe LS. Duplication of the vertebro-basilar system. Australas Radiol. 1991; 35(3): 220-224, doi: 10.1111/j.1440-1673.1991.tb03012.x, indexed in Pubmed: 1763984.

22. Trivelato FP, Abud DG, Nakiri GS, et al. Basilar artery fenestration aneurysms: endovascular treatment strategies based on 3D morphology. Clin Neuroradiol. 2016; 26(1): 73-79, doi: 10.1007/s00062-014-0336-0, indexed in Pubmed: 25164692.

23. Weis J, Reul J, Mayfrank L, et al. Duplication of a vertebral artery associated with epidermoid cyst of the posterior fossa. Eur Radiol. 1997; 7(3): 412-414, doi: 10.1007/ s003300050177, indexed in Pubmed: 9087368.

24. Yoon S, Chun Y, Kwon Y, et al. Vertebrobasilar junction aneurysms associated with fenestration: experience of five cases treated with guglielmi detachable coils. Surgical Neurology. 2004; 61(3): 248-254, doi: 10.1016/s00903019(03)00485-3.

25. Zhu DY, Fang YB, Wu YN, et al. Treatment of fenestrated vertebrobasilar junction-related aneurysms with endovascular techniques. J Clin Neurosci. 2016; 28: 112-116, doi: 10.1016/j.jocn.2015.09.018, indexed in Pubmed: 26778513. 